\title{
Measuring the shape: performance evaluation of a photogrammetry improvement applied to the Neanderthal skull Saccopastore 1
}

\author{
Costantino Buzi ${ }^{1}$, lleana Micarelli ${ }^{2}$, Antonio Profico ${ }^{1}$, Jacopo Conti ${ }^{3}$, Roberto Grassetti ${ }^{4}$, Walter \\ Cristiano ${ }^{5}$, Fabio Di Vincenzo ${ }^{4}$, Mary Anne Tafuri ${ }^{1}$, Giorgio Manzi ${ }^{1}$ \\ ${ }^{1}$ Dipartimento di Biologia Ambientale, Sapienza Università di Roma, Rome, Italy \\ 2 Dipartimento di Scienze dell'Antichità, Sapienza Università di Roma, Rome, Italy \\ ${ }^{3}$ Dipartimento di Scienze della Terra, Sapienza Università di Roma, Rome, Italy \\ ${ }^{4}$ Museo di Antropologia "Giuseppe Sergi", Rome, Italy \\ ${ }^{5}$ Dipartimento di Scienze biologiche, geologiche e ambientali - BIGEA, Università di Bologna, Bologna , Italy
}

\begin{abstract}
Several digital technologies are nowadays developed and applied to the study of the human fossil record. Here, we present a low-cost hardware implementation of the digital acquisition via photogrammetry, applied to a specimen of paleoanthropological interest: the Neanderthal skull Saccopastore 1. Such implementation has the purpose to semi-automatize the procedures of digital acquisition, by the introduction of an automatically rotating platform users can easily build on their own with minimum costs. We provide all the technical specifications, mostly based on the Arduino UNO ${ }^{\text {TM }}$ microcontroller technology, and evaluate the performance and the resolution of the acquisition by comparing it with the CT-scan of the same specimen through the calculation of their shape differences. In our opinion, the replication of the automatic rotating platform, described in this work, may contribute to the improvement of the digital acquisition processes and may represent, in addition, a useful and affordable tool for both research and dissemination.
\end{abstract}

\section{Section: RESEARCH PAPER}

Keywords: 3D-imaging; digital acquisition; geometric morphometrics; paleoanthropology; virtual anthropology

Citation: Costantino Buzi, lleana Micarelli, Antonio Profico, Jacopo Conti, Roberto Grassetti, Walter Cristiano, Fabio Di Vincenzo, Mary Anne Tafuri, Giorgio Manzi, Measuring the shape: performance evaluation of a photogrammetry improvement applied to the Neanderthal skull Saccopastore 1, Acta IMEKO, vol. 7, no. 3, article 13, October 2018, identifier: IMEKO-ACTA-07 (2018)-03-13

Section Editor: Sabrina Grassini, Politecnico di Torino, Italy

Received April 20, 2018; In final form September 21, 2018; Published October 2018

Copyright: (๑ 2018 IMEKO. This is an open-access article distributed under the terms of the Creative Commons Attribution 3.0 License, which permits unrestricted use, distribution, and reproduction in any medium, provided the original author and source are credited

Corresponding author: Costantino Buzi, costantino.buzi@uniroma1.it

\section{INTRODUCTION}

Since digital technologies started to be applied to specimens of paleontological or archaeological interest, there has been a great improvement, from both quantitative and qualitative point of views, of the information extractable from a single object of study [1]. This has happened mainly thanks to the development of virtual models of the actual specimens, which allow many researchers around the world to access unique remains or artifacts with no risk of damages and less difficulties in general [2], [3], [4].

Digital X-ray based acquisitions allow to observe the specimens with minimum or no handling at all, up to highly detailed level, also in the internal portions not accessible in the actual object [5], [6], [7], [8]. Apart from this scientific major achievement, a related advantage for the scientific community is the increase of the data-sharing policy for such virtual specimens, which is topped by the availability of online databases (e.g. NESPOS, Morphosource, Digimorph, Morphomuseum, Kupri).

In palaeoanthropology, the most important and renowned specimens are indeed of inestimable value and thus their access is more difficult. Thus, the diffusion of virtual anthropology protocols and techniques facilitated the spreading of the research on human fossils and the production of a new type of casts coming from the digital specimens via rapid prototyping (the more and more common 3D printing) [9], [10].

Notwithstanding the diffusion of such techniques also for purpose as study, displaying or dissemination [11], [12], many of 
the actual objects remain nevertheless of limited availability to subjects interested to them, while a good quality cast can be of considerable cost.

To overcome such difficulties there has been an increase in the use of acquisition and printing procedures less demanding from the perspectives of both costs and needed equipment [13]. The most commonly used is photogrammetry, which relies mainly on the performance of one or more series of photos (arranged in chunks) of the object in different norms [14]. The chunks are subsequently assembled via a dedicate software (e.g. Agisoft Photoscan) to eventually obtain a virtual volume (mesh) reporting the visible details and texture of the object.

\subsection{Digital acquisition}

Virtual anthropology is based on the study of the digital acquisition of human remains. The methods commonly used to digitally acquire an object are Computerized Tomography (CT), laser scanning and photogrammetry. Among the three, the CT scan is the acquisition procedure which returns both the inner and outer structures of the object, while both laser scan and photogrammetry only return its external topology, including colours and texture [15].

CT scan works by $\mathrm{X}$ rays, coming from a rotating source, passing through the object to be scanned at the centre of the rotation axis, to reach a series of sensor located on the opposite side of the source. The different densities of the object attenuate the $\mathrm{X}$ radiation and the output of the acquisition is a series of cross-sections of the object (slices) spaced out by a distance (inter-slice) which determines the final resolution. The slices are actually 2D images, useful for the medical purposes of the common CT scans. To obtain a 3D output, thus a polygonal mesh, the $2 \mathrm{D}$ images have to be processed, by applying the "Marching cubes" algorithm, after the definition of a value of radio density (expressed as Hounsfield unit) [2], [16], [17].

To be carried out, a CT acquisition requires the availability of professional medical equipment and space. Alternatively, a proper medical structure or research institution where the specimen to be scanned has to be carried, with consequent expenses for transportation and insurance, is needed.

The laser scanner acquisition relies on the triangulation built by a structured light beam intercepting the surface of the specimen and being detected by a sensor put at a known distance from the source [18], [19]. Laser scanner technology can return very highly detailed reproductions of the external surface of the objects: the quality of the acquisition depends on the hardware of the devices used, directly proportional to their cost, often expensive. No inner structures or details can be detected by this type of acquisition technology.

Photogrammetry is the less expensive acquisition procedure and is based on the assemblage of one or more series of images of the object, in its different views [20]. The images are usually photos taken by an operator and commonly grouped in different chunks, one for each norm. A neutral background and an optimal lighting, constant for the entire photo session, contribute significantly to a high-quality final output. The photos, exported to a dedicated software (e.g. Agisoft Photoscan), are aligned to obtain a sparse point cloud and subsequently a dense point cloud and a 3D mesh is built through a triangulation algorithm [21] The information on texture and colour is comprised in the final reconstruction, while a metric reference is needed, as photogrammetry, unlike CT and laser scans, does not return the object at the original scale [22], [23]. Not including the costs of a photographic equipment, the only added cost is that of a commercial software, as Agisoft, for the processing of the images, although several open source alternatives are available (e.g. insight3dng, Regard3D, MeshRecon and others).

Even though photogrammetry is a low-cost acquisition procedure, lacks a similarly cheap automation and can be significantly time consuming both from the point of view of the photoshoot, to be performed by an operator, and of the subsequent processing of images. In addition, there is no instant feedback on the output.

\subsection{Purpose}

The main purpose of this work, apart from presenting a hardware implementation we designed, is to evaluate an automated procedure of photogrammetry and demonstrate that the resulting output can substitute that of a more expensive and demanding procedure, as the CT scan, whenever the latter is not possible. Moreover, we demonstrate through a morphological analysis the feasibility of a good photogrammetry acquisition for research purposes as well as dissemination and education ones.

The hardware improvement we present in this paper is designed to overcome the need of manually performing the photoshoot, to stabilize the process, allowing the operator, at the same time, to perform other tasks such as the import of photos, the preparation of other specimens or the check for the alignment of previous photo sessions.

\subsection{Summary}

In the next section, we first point at the chosen case study, the renowned Neanderthal skull Saccopastore 1 from Rome, Italy; subsequently we present the technical specifications of the micro-CT scan used as comparison and the equipment used for the photogrammetry, with a focus on the hardware and the rotating platform; lastly, we point at the methods used for the sampling and the analysis. In the last section, we present both the digital reconstructions of Saccopastore 1 and discuss the results of the analysis.

\section{MATERIALS AND METHODS}

\subsection{Saccopastore 1}

Saccopastore 1 (SCP1) is a nearly complete cranium coming from a Neanderthal individual of remarkable antiquity, with a debated dating estimated to be between 100 - $130 \mathrm{ka}$ [24], and $250 \mathrm{ka}$ [25]. The specimen, lacking the mandible and bearing some taphonomic damages, as the missing maxillary processes and part of dentition, probably comes from a female individual and appears nonetheless well preserved [26], despite some damages occurred at the moment of the discovery (especially to the whole supraorbital torus and the vault) in April 1929.

The cranium, after its discovery in a gravel quarry in the Rome's countryside, today corresponding with the present city's suburbs, was brought by the quarry's owner to the anthropologist Sergio Sergi, who recognized and described its Neanderthal features, such as the mid-facial prognathism and the general shape of the cranial vault [27]. The skull is today preserved at the 'G. Sergi' Anthropology Museum of Sapienza University of Rome. 

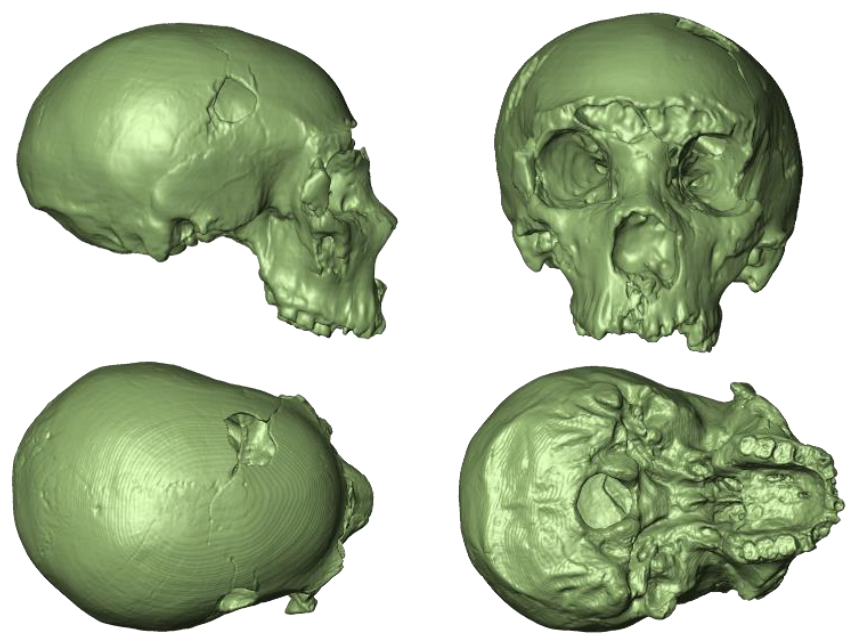

Figure 1. Digital model of SCP1, acquired via Computerized Tomography.

\subsection{Acquisition of the specimen}

SCP1 has been CT scanned using a Tomoscan AUEP (Philips), with an interslice distance equals to $1 \mathrm{~mm}$ scans. Data were exported as DICOM files, with a matrix of $512 \times 512$ pixels. SCP1 was scanned at $75 \mathrm{~mA}$ and $140 \mathrm{kV}$ (scantime $=4 \mathrm{~s}$ ), with a FOV of $250 \mathrm{~mm}$ and a consequent pixel size of $0.49 \mathrm{~mm}$ [28] (Figure 1).

The close-range photogrammetry of the same specimen was performed at the 'G. Sergi' Anthropology Museum of Sapienza University of Rome, by using a reflex camera Canon EOS 700D, with a Canon Zoom Lens $18-55 \mathrm{~mm}$, set to autofocus with ISO on 100, F-stop at $\mathrm{f} / 10$ and exposing time to $1 / 2 \mathrm{sec}$. The photos have been exported as .JPG files with $5184 \times 3456$ pixels resolution at $72 \mathrm{dpi}$.

The camera was set up on a Manfrotto tripod at a distance of about $45 \mathrm{~cm}$ from the specimen, located in a Havox Lightbox
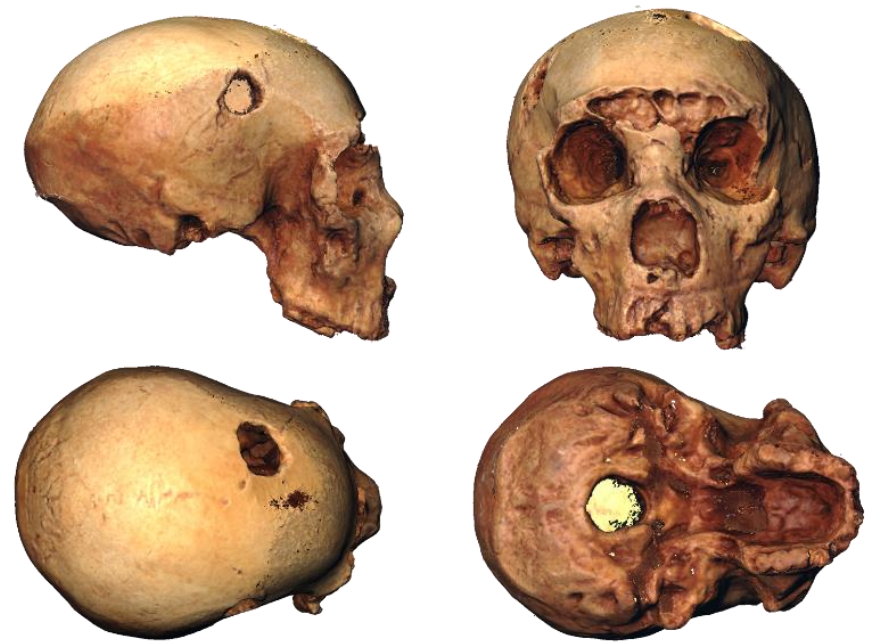

Figure 2. Digital model of SCP1, acquired via Photogrammetry.

$60 \times 60 \times 60 \mathrm{~cm}$. Four chunks were performed: the first was carried out by positioning SCP1 on the Frankfurt horizontal, right in front of the camera; the same position was kept for the second one, but positioning the camera $30^{\circ}$ above the horizontal, and subsequently, SCP1 was positioned upside down, resting on its bregmatic area, for the third chunk; eventually, for the fourth chunk, SCP1 was positioned on its occipital squama. Each chunk consisted of 50 photos, separated by 240 angular steps (more on it in section 2.3).

The four chunks thus acquired were processed by using the software Agisoft Photoscan ${ }^{\mathrm{TM}}$ (v. 1.2.6.2834). First, Agisoft allowed to remove the background from the images by using a mask; then, by using the metadata associated with the Exchangeable Image file Format (Exif) [29] of the photos, the program extrapolated from the data the position and orientation

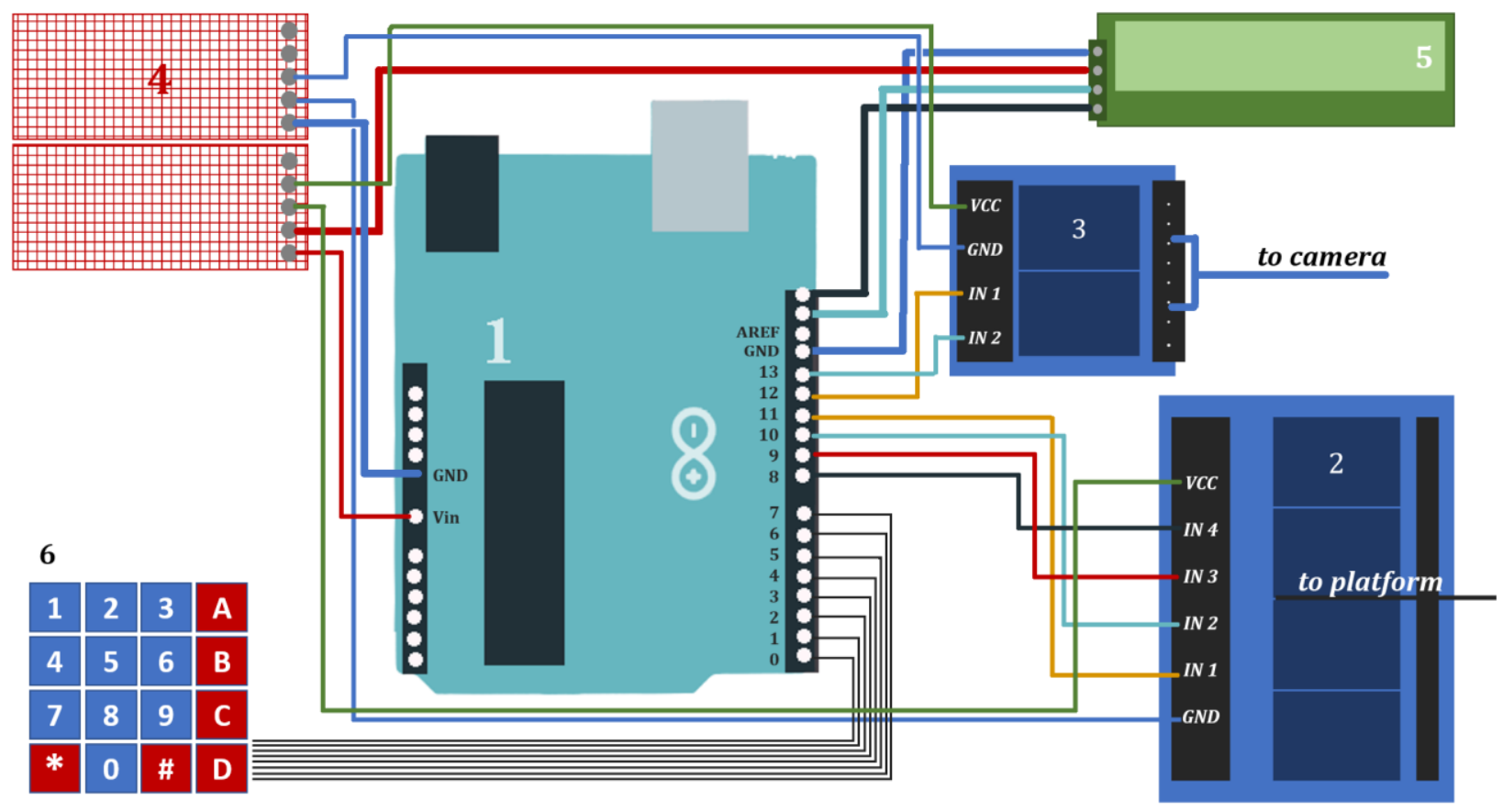

Figure 3. Scheme of the rotating platform system; the single modules are reported. 1: Microcontroller Arduino UNO ${ }^{\text {rM }}$ R3; $2: 4$ relay module connected with the step motor; 3: 2 relay module connected with the camera; 4: matrix board; 5: LC display 1602; 6: keypad. 
of the camera for each photo and built a first 3D point cloud. Subsequently, the program built a dense point cloud by estimating the depth information for each shot.

These steps were performed for each chunk and eventually all of them were aligned and combined by the software in a single dense cloud. The resulting mesh can be exported either in .PLY, .OBJ or.WRL format, among others (Figure 2).

The mesh obtained via photogrammetry was scaled to the real object size (cast of SCP1) by using the nasion-inion distance as reference.

\subsection{The rotating platform}

The system we designed is based on the Arduino UNO ${ }^{\mathrm{TM}}$ technology, a microcontroller board based on the ATmega328P microchip [30], [31], powered it with a AC-to-DC adapter (7.5 $12 \mathrm{~V} ; 2 \mathrm{~A})$. The microcontroller allows to synchronize the rotation of the platform with the focus and the photo shoot, via a step motor; among the further functions available, it is possible to link a beeper to signal the end of the shooting session.

Our system consists of the following modules: microcontroller Arduino UNO ${ }^{\mathrm{TM}}$ R3, a LC display 1602 (16 characters, 2 rows), a 4 relay module for operating the step motor, a 2 relay module for operating camera focus and shooting, a 4x4 keypad and a matrix board for the connections between modules; the latter is needed for the distribution of the voltage to the different modules from the microcontroller. The detailed scheme of the system is reported in Figure 3. The microcontroller has been programmed via Arduino Software ${ }^{\mathrm{TM}}$ 1.8.5; we provide the code in the Supplementary Information.

The system is run by entering the number of steps to take between each shoot, as indicated by the display. The number of photos decided by the operator is in relation with the number of steps needed by the step motor to perform a complete rotation: the more steps the platform can perform, the more precise is the system. The platform we used comes from a broken laser scanner and requires 12000 steps to perform a complete rotation. A calculation (1) is needed to obtain the requested number of steps for each shoot:

\section{steps for a complete rotation}

number of photo shoots

Thus, for performing a set of 50 photos, the system is programmed by entering first the number of steps required (e.g. $12000 / 50=240$ ); subsequently, the 'A' key (Figure 2) is pressed to accept the entry and the number of shoots requested (e.g. 50) is entered, followed by the '\#' key to run the program. To stop and reset the program, if needed, the key to enter is the '*' (asterisk).

\subsection{Sampling}

As stated before, we used a CT-scan of SCP1 to evaluate the precision of the model built via photogrammetry.

For the comparison between the two digital reconstructions of SCP1 we used a landmark-based, geometric morphometrics approach [32], [33]. Geometric morphometrics allows a quantitative analysis of the shape of the objects of study by using sets of homologous points, called landmarks [34]; the sets, or landmarks configurations, are eventually compared by excluding the effects of their size, orientation and position in the space by the Generalized Procrustes Analysis (GPA) [35].

The chosen landmark set for the comparation is represented in Figure 4. Once the landmark configurations have been sampled by the 3D imaging software Thermo Scientific Amira ${ }^{\mathrm{TM}}$ (version 5.4.5) on the two 3D models, their mesh distance [36] was evaluated by an analysis performed in $\mathrm{R}$ statistical environment [37], package 'Morpho' [38]. The mesh distance calculates the distance from the reference to the target $3 \mathrm{D}$ models, by projecting each vertex of the reference mesh (obtained by photogrammetry) to the target mesh (obtained by CT-scan).

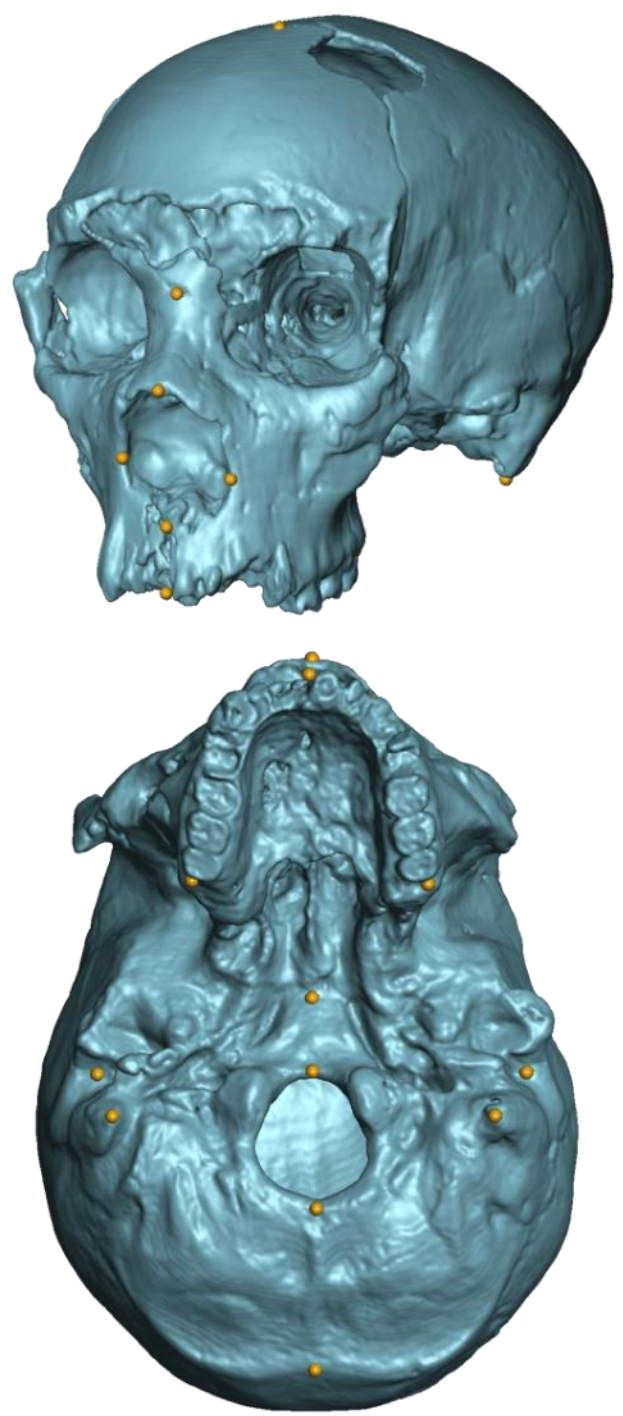

Figure 4. Landmark configuration used for the shape analysis.

\section{RESULTS}

The same landmark configuration was defined and acquired on both models of SCP1. The definition of a homologous landmark configuration allowed to obtain a rotation matrix (via GPA, [39]) in order to align the two 3D models. The Procrustes distance between the two landmark configurations is equal to $53.83 \mathrm{~mm}$, while the Euclidean distance amounts to $27.48 \mathrm{~mm}$. The landmark displacement is ranged from 0.43 to $3.08 \mathrm{~mm}$ with a mean displacement equal to $1.6 \mathrm{~mm}$.

The mesh distance measured and mapped the vertex displacement from the reference mesh (SCP1 via photogrammetry) to the target mesh (SCP1 via CT-scan). The mean vertex displacement is equal to $0.047 \mathrm{~mm}$ and the first and the third quantiles amount to -0.789 and $1.243 \mathrm{~mm}$ respectively. 
The results are represented in Figure 5, reporting the vertex displacement between the two models. In Figure 6, the mesh distance between the two models is graphically displayed.

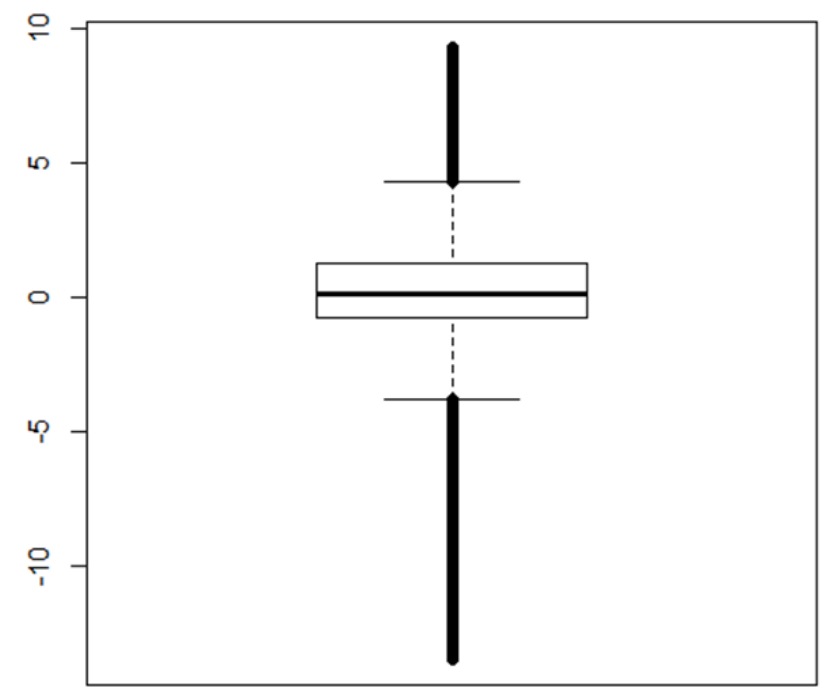

Figure 5. Boxplot of the vertex displacement $(\mathrm{mm})$ obtained after the mesh distance computing.

\section{CONCLUSIONS}

In recent years, several methods aimed at the digital acquisition were developed leading to an increase of technologies applied to the study of the human fossil record and cultural heritage. The use of the photogrammetry led to an increase in the sharing of human remains, by hosting them into virtual museums such as Nespos (www.nespos.org), Morphosource (morphosource.org) and AfricanFossils (africanfossils.org).

The 3D models acquired by digital methods can be used both for research and dissemination purposes.

This contribution presented the photogrammetric surveying and 3D modelling of the Neanderthal skull of Saccopastore 1, one of the most important and complete Neanderthal skulls ever discovered, characterized by a challenging texture, a complex shape/geometry and difficult optical properties (e.g. reflectivity).

We described in detail how to build, set up and use a rotating platform connected to an Arduino $\mathrm{UNO}^{\mathrm{TM}}$ microcontroller to speed-up and automatize the process of photogrammetry, as well as the procedures to correctly launch it.

The model obtained by our acquisition resulted complete and detailed, with the anatomical traits fully recognizable and feasible for the definition of landmarks and/or linear measurements. In contrast to other expensive or more demanding approaches, the method of close-range photogrammetry of SCP1, here presented, can be evaluated as a cutting edge effective, low-cost and automated technique.

The reproducibility of the model was evaluated by comparison with a CT scan of the same specimen. The anatomical distance both in terms of landmark and vertex displacement is low, indicating an almost full overlap of the two 3D models.

The only structures presenting appreciable differences between the values of vertex distance resulted to be 'grey' areas, not easy to acquire. The resulting topological artifacts are easy to fix by using specialized software (e.g., Geomagic Studio and Zbrush). Alternatively, the speeding up of the whole process allows to possibly include preliminary test sessions for making adjustments on aspects as the camera setting.

In fact, a crucial point of the process is based on finding the perfect setting of the Reflex. By setting the lowest value of ISO (100) the quality of the images we obtained still showed a very fine grain. Moreover, the F-stop at f/10 determined a good balance between the ratio of the system's focal length and the diameter of the entrance of the light for the set into the Lightbox. These two settings guaranteed the high-quality of the photos.

The use of the automatic rotating platform as shown in this work could represent an improvement highly facilitating research and dissemination for the cost-effectiveness of both hardware and software and fast and reproducible protocols for the digitalization of human remains.
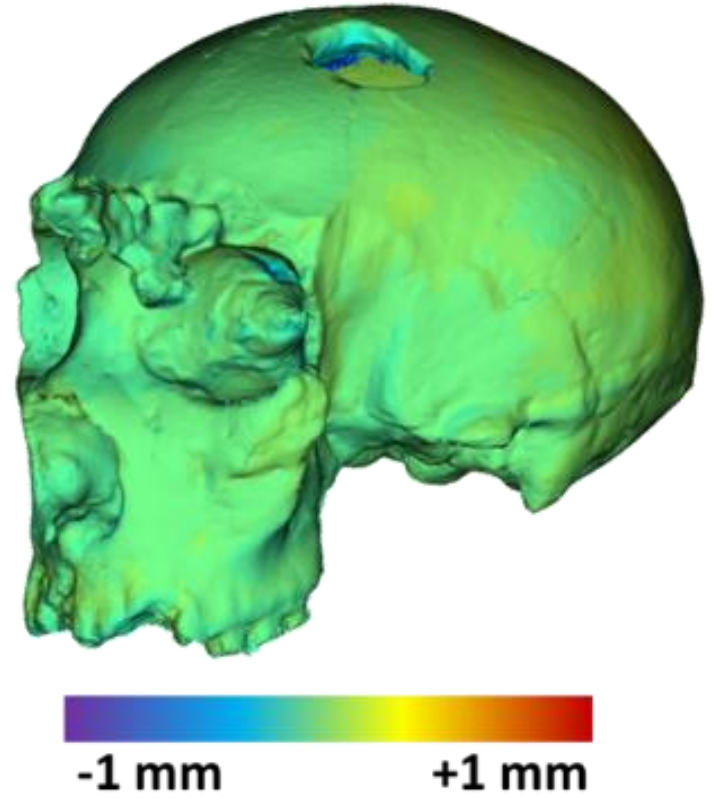

Figure 6. On the left, the overlapped models of SCP1 (white: CT-scan; yellow: photogrammetry), aligned after GPA. On the right, the mesh distance performed between the two 3D models. 


\section{ACKNOWLEDGEMENT}

We thank the "G. Sergi" Museum of Anthropology of Sapienza University of Rome for making available the used materials.

We want to thank, in addition, the Committee of the International Workshop on Metrology for Archaeology and Cultural Heritage (MetroArcheo).

\section{REFERENCES}

[1] A. Profico, L. Bellucci, C. Buzi, F. Di Vincenzo, I. Micarelli, F. Strani, M. A. Tafuri, and G. Manzi, Virtual Anthropology and its Application in Cultural Heritage Studies, Studies in Conservation, Sep. 2018.

[2] G. W. Weber, Virtual anthropology (VA): A call for Glasnost in paleoanthropology, Anat. Rec., vol. 265, no. 4, pp. 193-201, Aug. 2001.

[3] G. W. Weber, Another link between archaeology and anthropology: Virtual anthropology, Digit. Appl. Archaeol. Cult. Herit., vol. 1, no. 1, pp. 3-11, Jan. 2014.

[4] R. Scopigno, P. Cignoni, N. Pietroni, M. Callieri, and M. Dellepiane, Digital Fabrication Techniques for Cultural Heritage: A Survey, Comput. Graph. Forum, vol. 36, no. 1, pp. 6-21, Jan. 2017.

[5] F. W. Zonneveld, C. F. Spoor, J. Wind, The use of the CT in the study of the internal morphology of hominid fossils, MedicaMundi, 34 pp. 117-128., Dec. 1989.

[6] P. Wilson et al., Utilizing X-ray computed tomography for heritage conservation: The case of Megalosaurus bucklandii, in I2MTC 2017 - 2017 IEEE International Instrumentation and Measurement Technology Conference, Proceedings, 2017, pp. 1-5.

[7] X. Ni, J. J. Flynn, A. R. Wyss, Imaging the inner ear in fossil mammals: High-resolution CT scanning and $3-\mathrm{D}$ virtual reconstructions, Palaeontol. Electron., vol. 15, no. 2, 2012.

[8] A. Profico, S. Schlager, V. Valoriani, C. Buzi, M. Melchionna, A. Veneziano, P. Raia, J. Moggi-Cecchi, G. Manzi, Reproducing the internal and external anatomy of fossil bones: Two new automatic digital tools, Am. J. Phys. Anthropol., vol. Apr. 2018.

[9] C. P. E. Zollikofer, M. S. Ponce de León, Tools for Rapid Prototyping in the Biosciences, IEEE Comput. Graph. Appl., vol. 15, no. 6, pp. 48-55, 1995.

[10] R. Parry, Museums in a Digital Culture. 2016.

[11] D. B. Short, Use of 3D Printing by Museums: Educational Exhibits, Artifact Education, and Artifact Restoration, 3D Print. Addit. Manuf., vol. 2, no. 4, pp. 209-215, Dec. 2015.

[12] M. Neumüller, A. Reichinger, F. Rist, and C. Kern, 3D Printing for Cultural Heritage: Preservation, Accessibility, Research and Education, in 3D Research Challenges, Springer, Berlin, Heidelberg, 2014, pp. 119-134.

[13] J. De Reu et al., Towards a three-dimensional cost-effective registration of the archaeological heritage, J. Archaeol. Sci., vol. 40, no. 2, pp. 1108-1121, Feb. 2013.

[14] J. McCarthy, Multi-image photogrammetry as a practical tool for cultural heritage survey and community engagement, J. Archaeol. Sci., vol. 43, pp. 175-185, Mar. 2014.

[15] C. Nicolae, E. Nocerino, F. Menna, F. Remondino, Photogrammetry applied to problematic artefacts, Int. Arch. Photogramm. Remote Sens. Spat. Inf. Sci., p. XL-5: 451-456, 2014.

[16] P. F. Wilson, J. Stott, J. M. Warnett, A. Attridge,
M. P. Smith, M. A. Williams, Evaluation of Touchable 3DPrinted Replicas in Museums, Curator Museum J., vol. 60, no. 4, pp. 445-465, Oct. 2017.

[17] W. E. Lorensen, H. E. Cline, Marching cubes: A high resolution 3D surface construction algorithm, in Proc. of the 14th annual conference on Computer graphics and interactive techniques - SIGGRAPH '87, 1987, vol. 21, no. 4, pp. 163 169.

[18] L. Aiello, B. Wood, C. Key, C. Wood, Laser Scanning and Paleoanthropology, in Primate locomotion: recent advances, Boston, MA: Springer US, 1998, p. 223.

[19] M. Friess, L. F. Marcus, D. P. Reddy, E. Delson, The use of $3 \mathrm{D}$ laser scanning techniques for the morphometric analysis of human facial shape variation, BAR Int Ser., vol. 1049, pp. 31-5, 2002.

[20] E. P. Baltsavias, A comparison between photogrammetry and laser scanning, ISPRS J. Photogramm. Remote Sens., vol. 54, no. 2-3, pp. 83-94, Jul. 1999.

[21] D.-T. Lee, B. J. Schachter, Two algorithms for constructing a Delaunay triangulation, Int. J. Comput. Inf. Sci., vol. 9, no. 3, pp. 219-242, Jun. 1980.

[22] P. L. Falkingham, Acquisition of high resolution threedimensional models using free, open-source, photogrammetric software, Palaeontol. Electron., vol. 15, no. 1, pp. 1-15, 2012.

[23] G. Tembe, S. Siddiqui, Applications of computed tomography to fossil conservation and education, Collect. Forum, vol. 28, no. 1-2, pp. 47-62, Jan. 2014.

[24] E. Bruner, G. Manzi, Saccopastore 1: the earliest Neanderthal? A new look at an old cranium, in Neanderthals Revisited: New Approaches and Perspectives, Springer, Dordrecht, 2006, pp. 23-36.

[25] F. Marra, P. Ceruleo, B. Jicha, L. Pandolfi, C. Petronio, L. Salari, A new age within MIS 7 for the Homo neanderthalensis of Saccopastore in the glacio-eustatically forced sedimentary successions of the Aniene River Valley, Rome, Quat. Sci. Rev., vol. 129, pp. 260-274, Dec. 2015.

[26] E. Bruner, G. Manzi, Paleoneurology of an 'early' Neandertal: endocranial size, shape, and features of Saccopastore 1, J. Hum. Evol., vol. 54, no. 6, pp. 729-742, Jun. 2008.

[27] S. Sergi, La scoperta di un cranio del tipo di Neandertal presso Roma, vol. 28. Società romana di antropologia, 1929.

[28] G. Manzi, E. Bruner, S. Caprasecca, G. Gualdi, P. Passarello, CT-scanning and virtual reproduction of the Saccopastore Neandertal crania, Riv. di Antropol., vol. 79, pp. 61-72, 2001.

[29] JEITA, Exchangeable image file format for digital still cameras, pp. 1-154, 2002.

[30] Y. A. Badamasi, The working principle of an Arduino, in Proc. of the $11^{\text {th }}$ International Conference on Electronics, Computer and Computation, ICECCO 2014, 2014, pp. 1-4.

[31] A. D'Ausilio, Arduino: A low-cost multipurpose lab equipment, Behav. Res. Methods, vol. 44, no. 2, pp. 305-313, Jun. 2012.

[32] F. L. Bookstein, Biometrics, biomathematics and the morphometric synthesis, Bull. Math. Biol., vol. 58, no. 2, pp. 313-365, Mar. 1996.

[33] D. C. Adams, F. J. Rohlf, D. E. Slice, Geometric morphometrics: Ten years of progress following the 'revolution,' Ital. J. Zool., vol. 71, no. 1, pp. 5-16, Jan. 2004.

[34] F. L. Bookstein, Thin-Plate splines and the atlas problem for biomedical images, in Lecture Notes in Computer Science 
(including subseries Lecture Notes in Artificial Intelligence and Lecture Notes in Bioinformatics), vol. 511 LNCS, Berlin/Heidelberg: Springer-Verlag, 1991, pp. 326-342.

[35] P. Mitteroecker, P. Gunz, S. Windhager, K. Schaefer, A brief review of shape, form, and allometry in geometric morphometrics, with applications to human facial morphology, Hystrix, vol. 24, no. 1, 2013.

[36] S. Schlager, A. Profico, F. Di Vincenzo, G. Manzi, Retrodeformation of fossil specimens based on 3D bilateral semi-landmarks: Implementation in the $\mathrm{R}$ package 'Morpho,' PLoS One, vol. 13, no. 3, e0194073, Mar. 2018, https://doi.org/10.1371/journal.pone.0194073.

[37] R Development Core Team, R: A language and environment for statistical computing. R Foundation for Statistical Computing, R Foundation for Statistical Computing, Vienna, Austria. ISBN 3-900051-07-0, 2016.

[38] S. Schlager, Morpho and Rvcg-Shape Analysis in R: RPackages for Geometric Morphometrics, Shape Analysis and Surface Manipulations, in Statistical shape and deformation analysis, Elsevier, 2017, pp. 217-256.

[39] F. J. Rohlf, D. Slice, Extensions of the Procrustes Method for the Optimal Superimposition of Landmarks, Syst. Zool., vol. 39, no. 1, p. 40, Mar. 1990. 